\title{
Assessment of the Performance of Magnetic Resonance Imaging/Ultrasound Fusion Guided Prostate Biopsy against a Combined Targeted Plus Systematic Biopsy Approach Using 24-Core Transperineal Template Saturation Mapping Prostate Biopsy
}

\author{
Francis Ting, ${ }^{1,2}$ Pim J. Van Leeuwen, ${ }^{1,2}$ James Thompson, ${ }^{1,2,3}$ Ron Shnier, ${ }^{4}$ Daniel Moses, ${ }^{3,5}$ \\ Warick Delprado, ${ }^{6}$ and Phillip D. Stricker ${ }^{1,2}$ \\ ${ }^{1}$ St. Vincent's Prostate Cancer Centre, Darlinghurst, NSW 2010, Australia \\ ${ }^{2}$ Garvan Institute of Medical Research, The Kinghorn Cancer Centre, Darlinghurst, NSW 2010, Australia \\ ${ }^{3}$ School of Medicine, University of New South Wales, Kensington, NSW 2052, Australia \\ ${ }^{4}$ Southern Radiology, Randwick, NSW 2031, Australia \\ ${ }^{5}$ Spectrum Radiology, Randwick, NSW 2031, Australia \\ ${ }^{6}$ Douglas Hanly Moir Pathology, Macquarie Park, NSW 2113, Australia
}

Correspondence should be addressed to Francis Ting; francisting@gmail.com

Received 27 December 2015; Accepted 12 April 2016

Academic Editor: Bruce J. Trock

Copyright (C) 2016 Francis Ting et al. This is an open access article distributed under the Creative Commons Attribution License, which permits unrestricted use, distribution, and reproduction in any medium, provided the original work is properly cited.

\begin{abstract}
Objective. To compare the performance of multiparametric resonance imaging/ultrasound fusion targeted biopsy (MRI/US-TBx) to a combined biopsy strategy (MRI/US-TBx plus 24-core transperineal template saturation mapping biopsy (TTMB)). Methods. Between May 2012 and October 2015, all patients undergoing MRI/US-TBx at our institution were included for analysis. Patients underwent MRI/US-TBx of suspicious lesions detected on multiparametric MRI +/- simultaneous TTMB. Subgroup analysis was performed on patients undergoing simultaneous MRI/US-TBX + TTMB. Primary outcome was PCa detection. Significant PCa was defined as $\geq$ Gleason score (GS) $3+4=7$ PCa. McNemar's test was used to compare detection rates between MRI/US-TBx and the combined biopsy strategy. Results. 148 patients underwent MRI/US-TBx and 80 patients underwent MRI/US-TBx + TTMB. In the MRI/US-TBx versus combined biopsy strategy subgroup analysis $(n=80)$, there were $55 \mathrm{PCa}$ and 38 significant PCa. The detection rate for the combined biopsy strategy versus MRI/US-TBx for significant PCa was $49 \%$ versus $40 \%(p=0.02)$ and for insignificant PCa was $20 \%$ versus $10 \%(p=0.04)$, respectively. Eleven cases $(14 \%)$ of significant PCa were detected exclusively on MRI/US-TBx and 7 cases $(8.7 \%)$ of significant PCa were detected exclusively on TTMB. Conclusions. A combined biopsy approach (MRI/US-TBx + TTMB) detects more significant PCa than MRI/US-TBx alone; however, it will double the detection rate of insignificant PCa.
\end{abstract}

\section{Introduction}

The generally accepted diagnostic approach for prostate cancer $(\mathrm{PCa})$ involves performing random biopsies based on abnormal digital rectal examination (DRE) and prostate specific antigen (PSA) levels. However, this approach is limited by the fact that a negative DRE does not necessarily exclude PCa $[1,2]$ and although the risk of high-grade PCa increases with PSA level, there is no serum PSA threshold that can completely exclude significant PCa [3]. The drawbacks of the random systematic, template-based sampling approach are the potential for missing significant $\mathrm{PCa}$ and oversampling of insignificant PCa [4]. Furthermore, random biopsies underestimate tumour grade compared with radical prostatectomy (RP) $30-40 \%$ of the time $[5,6]$.

Targeted biopsy to suspicious lesions on multiparametric magnetic resonance imaging (mp-MRI) has emerged with 
the potential advantages of increased diagnostic accuracy and fewer cores. It allows the sensitivity and negative predictive value (NPV) of mp-MRI to be utilised with the real-time capabilities of transrectal ultrasound. There are a number of MRI-targeted biopsy methods, which include in-gantry/inbore MRI guided biopsy, MRI/ultrasound fusion targeted biopsy (MRI/US-TBx), and cognitive guided biopsy. Much of the rapidly growing body of literature on MRI-targeted biopsy has compared MRI/US-TBx to 10-12 core transrectal ultrasound guided biopsy (TRUSGB) and systematic reviews and meta-analysis have concluded that MRI/US-TBx detects more significant PCa [7, 8] and less insignificant PCa [9] compared with TRUSGB.

In order to improve standardisation of the reporting and interpretation of this continually growing body of literature, the standards of reporting for MRI-targeted biopsy studies (START) of the prostate were published in 2013 [10], written by an international working group. In addition, the European Society of Urogenital Radiology (ESUR) published the PI-RADS scoring system [11] in order to standardise the interpretation and reporting of prostate mp-MRI, and subsequently this scoring system has been validated in both primary and repeat biopsy cohorts [12-15].

Recent studies have utilised transperineal template saturation mapping biopsy (TTMB) as a reference test [16-18] in evaluation of MRI/US-TBx given the limited sensitivity and concordance with prostatectomy specimens of standard 10-12 core TRUSGB [19]. The consensus has been that the gold standard for cancer detection in primary biopsy is a combination of systematic and targeted cores. Our study aims to build on the current body of evidence by comparing the performance of MRI/US-TBx to that of a combined biopsy strategy (TTMB + MRI/US-TBx) in a meaningful way by adhering to START and PI-RADS guidelines.

\section{Methods}

2.1. Patient Selection and Study Design. Between May 2012 and October 2015, all patients undergoing MRI/US-TBx aged $>40$ years, with abnormal PSA or DRE, were included for retrospective study analysis. Patients underwent mp-MRI to identify regions suspicious for PCa followed by MRI/USTBx of suspicious lesions \pm simultaneous 24-core TTMB. Indications for MRI/US-TBx included previous negative biopsy, region of interest (ROI) on $\mathrm{mp}-\mathrm{MRI}<1.5 \mathrm{~cm}$, or ROI located in an unusual location not included in a standard TTMB template. A subgroup analysis was performed on all patients undergoing simultaneous MRI/US-TBx and TTMB. Informed consent was obtained from all patients and data collection performed as part of an approval from our institutional Human Research Ethics Committee (SVH File Number 12/231).

2.2. Imaging Protocol. All mp-MRIs were performed at 2 centres. Centre 1 used a 1.5 Tesla magnet (b-value 0$800 \mathrm{~s} / \mathrm{mm}^{2}$ ) and Centre 2 used a 3 Tesla magnet ( $b$-value 0 $\left.1500 \mathrm{~s} / \mathrm{mm}^{2}\right)$. Mp-MRI was performed using a standard MRI protocol described in [13]. Previous analysis has shown that there is no significant difference in diagnostic performance between these two centres [13]. All mp-MRIs were supervised and reported by subspecialised uroradiologists with at least 12 years of experience in $>3000$ cases each (RS and DM) following the standardised 5-point Prostate Imaging Reporting and Data System (PI-RADS) scale. Regions of interest (ROIs) were assigned a score of 1 to 5 for each parameter (T2WI, DCEI, and DWI) and then based on these individual parameter scores an overall impression ROI score was given. MRI-derived ROIs were assigned to twentyseven regions of each prostate according to the standardised prostate reporting scheme [20]. ROI size was measured as the maximal diameter as taken from axial slices on the mp-MRI.

2.3. Biopsy Methodology. TTMB and MRI/US-TBx were conducted by a single urologist (PS) who had full access to all mp-MRI data and was aware of the location of ROIs for both systematic and targeted biopsies. MRI/US-TBx was generally conducted first followed by TTMB.

2.3.1. TTMB. Transperineal, grid-directed template mapping biopsy was used as the reference test and was taken from 18 template locations using a modified Barzell technique [21]. A median of 24 cores were taken per patient and adjusted for volume with relative periurethral zone sparing.

2.3.2. MRI/US-TBx. MRI/transrectal ultrasound (TRUS) fusion biopsy was performed with a floor mounted, transperineal grid TRUS platform (BK Medical, Herlev, Denmark) combined with Biojet rigid MRI/TRUS fusion software (Meditron, Melbourne, Victoria, Australia). The biopsy operator had access to all mp-MRI data with radiologist marked ROIs in digital format. These ROIs were sampled under live MRI/TRUS fusion visualisation with at least 2 cores taken, adjusted for ROI size.

Targeted and systematic cores were labelled and reported separately by a single experienced uropathologist (WD).

2.4. Statistical Methods. Primary outcome was PCa detection (overall, significant, and insignificant). Significant PCa was defined as $\geq$ Gleason score (GS) $3+4=7$ PCa. Gleason scores were compared between MRI/US-TBx and TTMB. GS upgrading on MRI/US-TBx compared to TTMB was defined as significant when the change was from nil/insignificant $\mathrm{PCa}$ to significant PCa or from GS $3+4=7$ to $\geq \mathrm{GS} 4+3=7$.

McNemar's test was used to evaluate differences in detection rates of PCa between MRI/US-TBx, TTMB, and the combined biopsy strategy. Binary logistic multivariate regression analysis was used to assess the significance of ROI size in detection of significant PCa. Sensitivity, specificity, negative predictive value (NPV), and positive predictive value (PPV) of PI-RADS scoring system were calculated based on PI-RADS $1 / 2$ being negative and PI-RADS $3-5$ being positive, and the combined biopsy strategy was used as the gold standard. Statistical analyses were performed using SPSS ${ }^{\circledR}$ V21. A $p$ value $<0.05$ was considered statistically significant. 
TABLE 1: Baseline characteristics of MRI/US fusion targeted biopsy cohort.

\begin{tabular}{lcc}
\hline & All patients $(n=148)$ & Patients with both MRI/US-TBx + TTMB $(n=80)$ \\
\hline Age (median, IQR) & $65(60-70)$ & $64(59-69)$ \\
\hline PSA (median, IQR) & $5.0(3.6-7.5)$ & $5.2(3.8-7.5)$ \\
\hline Prostate volume (median, IQR) & $44(34-60)$ & $42(32-59)$ \\
\hline Positive DRE, $n(\%)$ & $23(16 \%)$ & $12(15 \%)$ \\
\hline Patients with previous biopsy, $n(\%)$ & $55(37 \%)$ & $15(19 \%)$ \\
Positive biopsy in the past, $n(\%)$ & $24(16 \%)$ & $8(10 \%)$ \\
$\quad$ Negative biopsy in the past, $n(\%)$ & $34(23 \%)$ & $7(9 \%)$ \\
\hline ROI size in mm & $7(5-10)$ & $7(5-10)$ \\
\hline Number of cores taken per location (Median, IQR) & $4(3-5)$ & $4(3-4)$ \\
\hline
\end{tabular}

$\mathrm{IQR}=$ interquartile range, $n=$ number, $\mathrm{mm}=$ millimetres, MRI/US-TBx $=\mathrm{MRI} /$ transrectal ultrasound fusion targeted biopsy, and TTMB $=$ transperineal template guided mapping biopsy.

TABLE 2: PI-RADS score and cancer detection rates $(n=148)$.

\begin{tabular}{lccc}
\hline \multirow{2}{*}{ PI-RADS } & \multirow{2}{*}{ Frequency } & \multicolumn{2}{c}{ MRI-fusion biopsy } \\
& & All PCa & Sig PCa \\
\hline $1 / 2^{*}$ & 28 & $4(14 \%)$ & $2(7 \%)$ \\
3 & 80 & $30(38 \%)$ & $17(21 \%)$ \\
4 & 55 & $38(69 \%)$ & $33(60 \%)$ \\
5 & 22 & $20(91 \%)$ & $18(82 \%)$ \\
Total & 185 & 92 & 70 \\
\hline
\end{tabular}

PCa $=$ prostate cancer, Sig $=$ significant.

${ }^{*}$ Due to clinical reasons these patients with PI-RADS 1/2 ROIs underwent targeted biopsy.

\section{Results}

3.1. MRI/US-TBx Patient Cohort. Demographic and baseline characteristics of the 148 included patients can be found in Table 1. Of these 148 patients, 93 (63\%) were undergoing primary biopsy and 55 (37\%) were undergoing repeat biopsy with $24(16 \%)$ patients having a previous positive biopsy. 185 ROIs were targeted in these 148 men. There were 28 (15\%) lesions scored as PI-RADS 1 or 2, 80 (43\%) as PI-RADS 3, 55 (30\%) as PI-RADS 4 and 22 (12\%) as PI-RADS 5 (Table 2). An increasing PI-RADS score correlated with an increasing $\mathrm{PCa}$ detection rate with the detection rate of significant $\mathrm{PCa}$ ranging from $7 \%$ for PI-RADS $1 / 2$ ROIs up to $82 \%$ for PIRADS 5 ROIs. Overall, there were 92 cancers detected with 22 (24\%) of these being GS $3+3=6$ and 70 (76\%) of these being GS $3+4=7$ or greater.

ROI size data is displayed in Table 3. For ROIs $<10 \mathrm{~mm}$, the detection rate of significant PCa on MRI/US-TBx was $29 \%$ versus $56 \%$ for ROIs $10 \mathrm{~mm}$ or greater. Overall detection rate of PCa on MRI/US-TBx was $43 \%$ versus $62 \%$ for ROIs $<10 \mathrm{~mm}$ versus ROIs $10 \mathrm{~mm}$ or greater, respectively. On multivariate analysis including age, PSA, DRE, primary versus repeat biopsy, prostate volume and PI-RADS score, ROI size was not significant $(p=0.09)$ but increasing age $(p=0.001)$ and PI-RADS score $\geq 4(p=0.02)$ were predictive in detection of significant PCa.
TABLE 3: Detection rate MRI/US fusion targeted biopsy by size of ROI $(n=148)$.

\begin{tabular}{|c|c|c|c|}
\hline \multirow{2}{*}{ Lesion size } & \multirow{2}{*}{ Freq. } & Any PCa & Sign PCa \\
\hline & & \multicolumn{2}{|c|}{ MRI/US Fusion targeted biopsy } \\
\hline$<5 \mathrm{~mm}$ & 22 & $11(50 \%)$ & $7(32 \%)$ \\
\hline $5-9 \mathrm{~mm}$ & 93 & $39(42 \%)$ & $26(28 \%)$ \\
\hline$\geq 10 \mathrm{~mm}$ & 45 & $28(62 \%)$ & $25(56 \%)$ \\
\hline
\end{tabular}

Freq. $=$ frequency, $\mathrm{PCa}=$ prostate cancer, Sign $=$ significant, and $\mathrm{ROI}=$ region of interest.

3.2. Comparison of Cancer Detection Performance: MRI/USTBx versus Combined Biopsy Strategy. Eighty patients underwent both MRI-TBx and TTMB simultaneously. In this subgroup analysis, 65 (81\%) patients underwent primary biopsy and 15 (19\%) underwent repeat biopsy. Baseline characteristics of this subgroup can be found in Table 1.

There were 55 (69\%) patients diagnosed with PCa and $38(45 \%)$ of these were significant PCa. The detection rate of significant PCa for MRI/US-TBx and combined biopsy strategy was $40 \%$ versus $49 \%(p=0.02)$ and the detection rate of insignificant PCa for MRI/US-TBx and combined biopsy strategy was $10 \%$ versus $20 \%$ ( $p=0.04)$, respectively (Table 4 ). The proportion of detected cancers being significant for each biopsy technique was 32/40 (80\%) for MRI/USTBx and 39/55 (71\%) for combined biopsy strategy (Table 4). Gleason score distribution for different biopsy techniques is displayed in Table 5.

Sixteen $(20 \%)$ patients had a GS upgrade on combined biopsy strategy compared to their MRI/US-TBx with seven (9\%) being significant upgrades. These missed cases are described in Table 6 . All cancers were Gleason $3+4=7 \mathrm{PCa}$ with a maximum of $15 \%$ Gleason 4 . In addition, the location of the significant cancers detected on TTMB but missed on MRI/US-TBx did not correlate to the location of the ROI on mp-MRI in five of these seven cases.

Sensitivity and negative predictive value (NPV) of PIRADS (when considering PI-RADS 1-2 negative, PI-RADS 35 positive) for detection of significant $\mathrm{PCa}$ were $98 \%$ and $92 \%$, 
TABLE 4: Detection rates for significant and insignificant prostate cancer of MRI/ultrasound fusion targeted biopsy versus transperineal template saturation mapping biopsy $(n=80)$.

\begin{tabular}{lccccc}
\hline & MRI/US-TBx & TTMB & $p$ value (MRI versus TTMB) & CBS & $p$ value (MRI versus CBS) \\
\hline Any prostate cancer, $n(\%)$ & $40(50 \%)$ & $48(60 \%)$ & 0.13 & $55(69 \%)$ & $<0.01$ \\
Significant prostate cancer, $n(\%)$ & $32(40 \%)$ & $28(35 \%)$ & 0.48 & $39(49 \%)$ & 0.02 \\
Insignificant prostate cancer, $n(\%)$ & $8(10 \%)$ & $20(25 \%)$ & 0.01 & $16(20 \%)$ & 0.04 \\
\hline
\end{tabular}

$\mathrm{MRI} / \mathrm{US}-\mathrm{TBx}=\mathrm{MRI} /$ transrectal ultrasound fusion targeted biopsy, TTMB $=$ transperineal template guided mapping biopsy, CBS = combined biopsy strategy (MRI/US-TBX + TTMB), and $n=$ number.

TABLE 5: Gleason score breakdown for MRI/US-TBx and TTMB $(n=80)$.

\begin{tabular}{|c|c|c|c|c|}
\hline Gleason score & MRI/US-TBx & TTMB & CBS & $p$ value (MRI/US-TBx versus CBS) \\
\hline No cancer & $40(50 \%)$ & $32(40 \%)$ & $25(31 \%)$ & \\
\hline $3+3$ & $8(10 \%)$ & $20(25 \%)$ & $16(20 \%)$ & \\
\hline $3+4$ & $18(22.5 \%)$ & $24(30 \%)$ & $25(31 \%)$ & \\
\hline $4+3$ & $7(9 \%)$ & $3(4 \%)$ & $7(9 \%)$ & \\
\hline $4+4$ & $4(5 \%)$ & $1(1 \%)$ & $4(5 \%)$ & \\
\hline $4+5$ & $2(2.5 \%)$ & 0 & $2(2.5 \%)$ & \\
\hline $5+4$ & $1(1 \%)$ & 0 & $1(1.5 \%)$ & \\
\hline Total & 80 & 80 & 80 & \\
\hline Low risk & $8(10 \%)$ & $20(25 \%)$ & $16(20 \%)$ & 0.04 \\
\hline Int. risk & $25(31 \%)$ & $27(34 \%)$ & $32(40 \%)$ & 0.02 \\
\hline High risk & $7(9 \%)$ & $1(1 \%)$ & $7(9 \%)$ & Not sig \\
\hline
\end{tabular}

CBS = combined biopsy strategy, MRI/US-TBx = MRI/transrectal ultrasound fusion targeted biopsy, and TTMB = transperineal template guided mapping biopsy.

Low risk $=$ GS 6, intermediate risk $=$ GS 7, and high risk $=$ GS 8 or greater.

respectively (Table 7). The PPV of PI-RADS for detection of significant PCa when considering PI-RADS $\geq 3, \geq 4$, and 5 as positive was $56 \%, 74 \%$, and $100 \%$, respectively.

3.3. Comparison of Cancer Detection Performance: MRI/US$T B x$ versus TTMB. Median number of cores taken per patient was 24 (IQR 21-29) for TTMB and 4 (IQR 3-4) for MRI/USTBx. Sampling efficiency was in favour of MRI/US-TBx with $20 \%$ of MRI/US-TBx versus $8 \%$ of TTMB cores detecting significant PCa.

The detection rate of significant $\mathrm{PCa}$ for MRI/US-TBx and TTMB was $40 \%$ versus $35 \%(p=0.48)$ and the detection rate of insignificant PCa for MRI/US-TBx and TTMB was $10 \%$ versus $25 \%(p=0.01)$.

Twenty patients (25\%) had a GS upgrade on MRI/USTBx compared to their TTMB with 15 (19\%) being significant upgrades. Eleven cases of significant PCa were detected exclusively on MRI/US-TBx and 7 cases of significant PCa (all Gleason $3+4=7$ with $\leq 15 \%$ Gleason 4 ) were detected exclusively on TTMB.

\section{Discussion}

Our results show that a biopsy strategy combining TTMB with MRI/US-TBx can increase the detection rate of significant PCa when compared to MRI/US-TBx alone; however this combined biopsy strategy also doubled the detection of insignificant PCa when compared to MRI/US-TBx alone. This is in line with recent data from a direct prospective comparison between MRI/US-TBx and TTMB which has shown that MRI/US-TBx can detect as many GS 7 or greater tumours while simultaneously avoiding the detection of $44 \%$ of lower grade disease [17]. Another series $(n=50)$ comparing both cognitive and software based MRI registration to TTMB has shown slightly lower rates of significant disease detection (68\% for software based fusion versus $76 \%$ for TTMB) [18], and similarly, in another series $(n=437)$ more significant PCa was found exclusively in TTMB than exclusively in MRI/US-TBx (52 versus 18 cases) [16]. Thus, if the aim is purely to maximise the detection of significant $\mathrm{PCa}$ the combination of TTMB with MRI/US-TBx still appears to be the most effective biopsy strategy at present. However, in the current series it should be noted that all additional significant cancers detected by the addition of TTMB to the MRI/US-TBx biopsy strategy were GS $3+4=7$ with a maximum GS 4 component of $15 \%$. Given the slow natural history and high 10-year PCa specific survival of GS $3+4$ PCa [22], a delayed diagnosis in the course of careful follow-up is unlikely to have resulted in missing the window of curability.

TRUSGB can miss $20-30 \%$ of clinically significant cancers due to undersampling, particularly in the anteroapical region of the prostate [23]. MRI/US-TBx overcomes this deficiency by sampling the suspicious region and thus is likely to be more effective than TRUSB in the detection of significant PCa. Accordingly, observational trials have shown that MRI/US-TBx has a higher detection rate for clinically significant cancer [24-26], a higher detection rate for high-risk PCa [27], and a lower sensitivity for clinically 
TABLE 6: Missed cancers (cancers detected on TTMB but missed on MRI targeted biopsy: significant upgrades only).

\begin{tabular}{|c|c|c|c|c|c|c|}
\hline TTM & IB data & & & JS fusion targeted bio & data & \\
\hline GS (\% Gleason 4) & Location of cancer & GS & Location of ROI & $\begin{array}{l}\text { MRI ROI location } \\
\text { correlates to TTMB }\end{array}$ & ROI size (mm) & PI-RAD score \\
\hline $3+4(15 \%)$ & $\mathrm{L}$ ant & 0 & L base post & No & 7 & 2 \\
\hline $3+4(5 \%)$ & $\mathrm{R}$ mid post & 0 & L post apex & No & 4 & 3 \\
\hline $3+4(10 \%)$ & L post apex & 0 & Midline post & No & 11 & 3 \\
\hline $3+4(10 \%)$ & $\mathrm{R}$ base $\mathrm{PL}+\mathrm{R}$ ant & 0 & $\mathrm{R}$ mid apex & No & 5 & 3 \\
\hline $3+4(10 \%)$ & $\mathrm{R}$ base PL & $3+3$ & $\mathrm{R}$ base peripheral zone & Partial & 12 & 3 \\
\hline $3+4(2 \%)$ & $\begin{array}{l}\mathrm{L} \text { ant apex, } \mathrm{R} \text { mid } \\
\text { PL, L mid PL }\end{array}$ & $3+3$ & L post apex & Partial & 9 & 4 \\
\hline $3+4(5 \%)$ & $\mathrm{R}$ base PL & 0 & L base & No & 2 & 5 \\
\hline
\end{tabular}

$\mathrm{MRI} / \mathrm{US}-\mathrm{TBx}=\mathrm{MRI} /$ transrectal ultrasound fusion targeted biopsy, TTMB $=$ transperineal template guided mapping biopsy, $\mathrm{ROI}=$ region of interest, $\mathrm{L}=$ left, $\mathrm{R}=$ right, ant $=$ anterior, post $=$ posterior, and $\mathrm{PL}=$ posterolateral .

TABLE 7: Sensitivity, specificity, NPV, and PPV of PI-RADS scoring system for prostate cancer detection, derived from combined biopsy strategy (MRI/US-TBx + TTMB). For this analysis PI-RADS $1 / 2$ is considered negative and PI-RADS $3-5$ is considered positive. $N=$ 80 .

\begin{tabular}{lccc}
\hline & All PCa & Sign PCa & Insig PCa \\
\hline Sensitivity & 96 & 98 & 91 \\
Specificity & 32 & 22 & 13 \\
NPV & 75 & 92 & 83 \\
PPV & 78 & 56 & 23 \\
\hline
\end{tabular}

$\mathrm{PCa}=$ prostate cancer, Sign $=$ significant, Insig $=$ insignificant, $\mathrm{NPV}=$ negative predictive value, and PPV = positive predictive value.

insignificant cancer compared to TRUSGB ( 0.51 versus 0.81 , resp.) [9]. In addition, MRI/US-TBx has been shown to be able to detect as many GS $\geq 7 \mathrm{PCa}$ compared to TTMB [17]. In the current series, we demonstrated a detection rate of MRI/US-TBx for significant PCa of $40 \%$. A number of significant cancers were missed by MRI/US-TBx but the majority of these were outside of the ROI targeted on mpMRI, which indicates that MRI/US-TBx is still a procedurally valid technique in our centre.

\subsection{Magnetic Resonance Imaging Targeted Biopsy Can Reduce} $P C a$ Overdiagnosis. The disadvantage of using TTMB alone is the increased detection of insignificant $\mathrm{PCa}$, with rates of insignificant PCa detection for TTMB of up to $25 \%$ being reported [28-30], which is consistent with the $25 \%$ rate of insignificant PCa detection by TTMB in the current series. Compared to this, MRI/US-TBx alone in the current series had a $10 \%$ detection rate of insignificant $\mathrm{PCa}$ and thus a future biopsy approach that replaces TTMB with MRI/US-TBx will significantly reduce the overdiagnosis of insignificant PCa.

4.2. Validation of the PI-RADS Scoring System. A PI-RADS score of $1 / 2$ appeared to be effective in ruling out most cases of significant PCa and a PI-RADS score of $4 / 5$ was able to predict prostate cancer; the data we present further validates the current data on the predictive information that is provided by PI-RADS scoring in prostate mp-MRI $[14,15]$. In patients with PI-RADS 3 "equivocal" ROIs there were a significant proportion (21\%) of patients with significant $\mathrm{PCa}$, and comparable rates of significant PCa (26-29\%) $[13,14]$ in PI-RADS 3 ROIs have been reported which supports the rationale of performing MRI-TBx on all patients with PIRADS 3-5 lesions on mp-MRI.

Centres of excellence have reported sensitivity of $86-96 \%$ $[13,14,31-34]$, and NPV of 90-92\% [14, 33, 35] for mp-MRI in detection of significant PCa. Our reported sensitivity and NPV of $98 \%$ and $92 \%$, respectively, is similar to these results and suggests the possibility of avoiding random biopsy in patients with negative mp-MRI, provided that standards are established for accreditation, training, and reporting of $\mathrm{mp}$ MRI. Lower sensitivities of $58-76 \%[36,37]$ and NPV of 79$89 \%[36,37]$ have been reported in smaller series and this interstudy variability can be part-attributed to the significant learning curve associated with interpretation of prostate mp-MRI of about 100 cases [38, 39]. However, interstudy variability of NPV is also influenced by the overall prevalence of cancer and needs to be taken into account when comparing studies.

4.3. Limitations. Firstly, the primary operator was not blinded to the ROI when performing random biopsies and therefore an accurate direct comparison between TTMB and MRI/US-TBx alone is not possible from this study given this confounding factor. However, we are still able to draw an accurate comparison between MRI/US-TBx versus the combined biopsy strategy, which gives this study clinical utility. Secondly, this study was retrospective in nature but as noted above we have strict, standardised mp-MRI and biopsy protocols in place. Thirdly, longer follow-up is required, particularly in the biopsy negative patients and our operator and 
radiologists were not blinded to radiological or clinical data as per routine clinical practice. Fourthly, there are inherent limitations in using biopsy as the reference test: MRI/US-TBx could have missed MRI targets despite software registration [40] and there is a percentage of upgrading that occurs on radical prostatectomy. However in this cohort using radical prostatectomy as the reference test was not feasible due to ethical issues and positive selection bias. Finally, the binary logistic regression analysis performed to assess significance of ROI size in detection of significant PCa was performed on a per-lesion rather than a per-patient basis. Some patients had multiple ROIs/lesions and the ensuing correlation effect was not accounted for with a mixed model analysis, which resulted in the $p$ values being more significant than they should be. However, this does not change the interpretation of nonsignificance for ROI size in detection of significant PCa.

4.4. Strengths. One of the main strengths of this study was the use of TTMB as a reference test. TTMB was used as it has been demonstrated as a robust reference test [13, 41], localises the index lesion [42] in most men with clinically significant disease [25], and detects more clinically significant cancer than 12-core TRUSGB [28]. Furthermore, another series demonstrated that in a significant proportion of men initially diagnosed with apparently low-risk disease on TRUSGB, subsequent TTMB revealed clinically significant PCa requiring more aggressive therapy [43]. Radical prostatectomy (RP), although the pathology gold standard reference test, could not be utilised in this study due to ethical considerations and positive selection bias.

\section{Conclusion}

A combined biopsy approach (MRI/US-TBx + TTMB) detects more significant PCa than MRI/US-TBx alone; however it will double the detection rate of insignificant PCa. MRI/US$\mathrm{TBx}$ is the likely future of PCa diagnosis and in centres of excellence is a robust and valid technique. It has the potential to increase the specificity of PCa detection and reduce overdiagnosis of insignificant PCa. However, further prospective trials with longer follow-up are required, particularly for biopsy negative cases.

\section{Competing Interests}

The authors declare that they have no competing interests.

\section{Acknowledgments}

Anne-Maree Haynes and Quoc Nguyen from Australian Prostate Cancer Research Centre-NSW (APCRC-NSW), IT Applications Group, and CANSTO Database are gratefully acknowledged. This research is supported by the Department of Health and Ageing for its funding of the Australian Prostate Cancer Research Centre-NSW, the National Health and Medical Research Council, St. Vincent's Prostate Cancer Centre, and the RT Hall Trust.

\section{References}

[1] C. Gosselaar, R. Kranse, M. J. Roobol, S. Roemeling, and F. H. Schröder, "The interobserver variability of digital rectal examination in a large randomized trial for the screening of prostate cancer," Prostate, vol. 68, no. 9, pp. 985-993, 2008.

[2] F. H. Schroder, P. van der Maas, P. Beemsterboer, A. B. Kruger, R. Hoedemaeker, J. Rietbergen et al., "Evaluation of the digital rectal examination as a screening test for prostate cancer. Rotterdam section of the European Randomized Study of Screening for Prostate Cancer," Journal of the National Cancer Institute, vol. 90, no. 23, pp. 1817-1823, 1998.

[3] I. M. Thompson, D. P. Ankerst, C. Chi et al., "Operating characteristics of prostate-specific antigen in men with an initial PSA level of $3.0 \mathrm{ng} / \mathrm{mL}$ or lower," The Journal of the American Medical Association, vol. 294, no. 1, pp. 66-70, 2005.

[4] O. Ukimura, J. A. Coleman, A. de la Taille et al., "Contemporary role of systematic prostate biopsies: Indications, techniques, and implications for patient care," European Urology, vol. 63, no. 2, pp. 214-230, 2013.

[5] A. V. D’Amico, A. A. Renshaw, L. Arsenault, D. Schultz, and J. P. Richie, "Clinical predictors of upgrading to gleason grade 4 or 5 disease at radical prostatectomy: potential implications for patient selection for radiation and androgen suppression therapy," International Journal of Radiation Oncology, Biology, Physics, vol. 45, no. 4, pp. 841-846, 1999.

[6] S. Scott, H. Samaratunga, C. Chabert, M. Breckenridge, and T. Gianduzzo, "Is transperineal prostate biopsy more accurate than transrectal biopsy in determining final Gleason score and clinical risk category? A comparative analysis," BJU International, vol. 116, supplement 3, pp. 26-30, 2015.

[7] M. Valerio, I. Donaldson, M. Emberton et al., "Detection of clinically significant prostate cancer using magnetic resonance imaging-ultrasound fusion targeted biopsy: a systematic review," European Urology, vol. 68, no. 1, pp. 8-19, 2015.

[8] J. Wu, A. Ji, B. Xie et al., "Is magnetic resonance/ultrasound fusion prostate biopsy better than systematic prostate biopsy? An updated meta- and trial sequential analysis," Oncotarget, vol. 6, no. 41, pp. 43571-43580, 2015.

[9] I. G. Schoots, M. J. Roobol, D. Nieboer, C. H. Bangma, E. W. Steyerberg, and M. G. M. Hunink, "Magnetic resonance imaging-targeted biopsy may enhance the diagnostic accuracy of significant prostate cancer detection compared to standard transrectal ultrasound-guided biopsy: a systematic review and meta-analysis," European Urology, vol. 68, no. 3, pp. 438-450, 2015.

[10] C. M. Moore, V. Kasivisvanathan, S. Eggener et al., "Standards of reporting for MRI-targeted biopsy studies (START) of the prostate: recommendations from an international working group," European Urology, vol. 64, no. 4, pp. 544-552, 2013.

[11] J. C. Weinreb, J. O. Barentsz, P. L. Choyke et al., "Pi-rads prostate imaging-reporting and data system: 2015, version 2," European Urology, vol. 69, no. 1, pp. 16-40, 2015.

[12] H. Cash, A. Maxeiner, C. Stephan et al., "The detection of significant prostate cancer is correlated with the Prostate Imaging Reporting and Data System (PI-RADS) in MRI/transrectal ultrasound fusion biopsy," World Journal of Urology, vol. 34, no. 4, pp. 525-532, 2016.

[13] J. E. Thompson, D. Moses, R. Shnier et al., "Multiparametric magnetic resonance imaging guided diagnostic biopsy detects significant prostate cancer and could reduce unnecessary biopsies and over detection: a prospective study," The Journal of Urology, vol. 192, no. 1, pp. 67-74, 2014. 
[14] J. E. Thompson, P. J. van Leeuwen, D. Moses et al., “The diagnostic performance of multiparametric magnetic resonance imaging to detect significant prostate cancer," The Journal of Urology, 2015.

[15] D. Portalez, P. Mozer, F. Cornud et al., "Validation of the European Society of Urogenital Radiology scoring system for prostate cancer diagnosis on multiparametric magnetic resonance imaging in a cohort of repeat biopsy patients," European Urology, vol. 62, no. 6, pp. 986-996, 2012.

[16] F. Distler, J. P. Radtke, C. Kesch et al., "Value of MRI/ultrasound fusion in primary biopsy for the diagnosis of prostate cancer," Der Urologe, vol. 55, no. 2, pp. 146-155, 2016.

[17] J. P. Radtke, T. H. Kuru, S. Boxler et al., "Comparative Analysis of transperineal template saturation prostate biopsy versus magnetic resonance imaging targeted biopsy with magnetic resonance imaging-ultrasound fusion guidance," The Journal of Urology, vol. 193, no. 1, pp. 87-94, 2015.

[18] M. Valerio, N. McCartan, A. Freeman, S. Punwani, M. Emberton, and H. U. Ahmed, "Visually directed vs. software-based targeted biopsy compared to transperineal template mapping biopsy in the detection of clinically significant prostate cancer," Urologic Oncology, vol. 33, no. 10, pp. 424.e9-424.e16, 2015.

[19] M. A. Bjurlin, H. B. Carter, P. Schellhammer et al., "Optimization of initial prostate biopsy in clinical practice: sampling, labeling and specimen processing," The Journal of Urology, vol. 189, no. 6, pp. 2039-2046, 2013.

[20] L. Dickinson, H. U. Ahmed, C. Allen et al., "Magnetic resonance imaging for the detection, localisation, and characterisation of prostate cancer: recommendations from a European consensus meeting," European Urology, vol. 59, no. 4, pp. 477-494, 2011.

[21] W. Barzell WIW and G. Andriole, Transperineal Template Guided Saturation Biopsy of the Prostate: Rationale, Indications and Technique. Urology Times, vol. 31, Andriole G. Transperineal Template Guided Saturation Biopsy of the Prostate, Rationale, 2003.

[22] J. L. Wright, C. A. Salinas, D. W. Lin et al., "Prostate cancer specific mortality and gleason 7 disease differences in prostate cancer outcomes between cases with gleason $4+3$ and gleason $3+4$ tumors in a population based cohort," The Journal of Urology, vol. 182, no. 6, pp. 2702-2707, 2009.

[23] B. Gershman, A. L. Zietman, A. S. Feldman, and W. S. McDougal, "Transperineal template-guided prostate biopsy for patients with persistently elevated PSA and multiple prior negative biopsies," Urologic Oncology: Seminars and Original Investigations, vol. 31, no. 7, pp. 1093-1097, 2013.

[24] P. Mozer, M. Rouprêt, C. Le Cossec et al., "First round of targeted biopsies using magnetic resonance imaging/ultrasonography fusion compared with conventional transrectal ultrasonography-guided biopsies for the diagnosis of localised prostate cancer," BJU International, vol. 115, no. 1, pp. 50-57, 2014.

[25] A. R. Rastinehad, B. Turkbey, S. S. Salami et al., "Improving detection of clinically significant prostate cancer: magnetic resonance imaging/transrectal ultrasound fusion guided prostate biopsy," The Journal of Urology, vol. 191, no. 6, pp. 1749-1754, 2014.

[26] M. M. Siddiqui, S. Rais-Bahrami, H. Truong et al., "Magnetic resonance imaging/ultrasound-fusion biopsy significantly upgrades prostate cancer versus systematic 12-core transrectal ultrasound biopsy," European Urology, vol. 64, no. 5, pp. 713719, 2013.
[27] M. M. Siddiqui, S. Rais-Bahrami, B. Turkbey et al., "Comparison of MR/ultrasound fusion-guided biopsy with ultrasoundguided biopsy for the diagnosis of prostate cancer," The Journal of the American Medical Association, vol. 313, no. 4, pp. 390-397, 2015.

[28] N. Bittner, G. S. Merrick, A. Bennett et al., "Diagnostic performance of initial transperineal template-guided mapping biopsy of the prostate gland," American Journal of Clinical Oncology, vol. 38, no. 3, pp. 300-303, 2015.

[29] T. Klatte, N. Swietek, G. Schatzl, and M. Waldert, “Transperineal template-guided biopsy for diagnosis of prostate cancer in patients with at least two prior negative biopsies," Wiener Klinische Wochenschrift, vol. 125, no. 21-22, pp. 669-673, 2013.

[30] N. Bittner, G. S. Merrick, W. M. Butler, A. Bennett, and R. W. Galbreath, "Incidence and pathological features of prostate cancer detected on transperineal template guided mapping biopsy after negative transrectal ultrasound guided biopsy," The Journal of Urology, vol. 190, no. 2, pp. 509-514, 2013.

[31] M. Abd-Alazeez, A. Kirkham, H. U. Ahmed et al., "Performance of multiparametric MRI in men at risk of prostate cancer before the first biopsy: a paired validating cohort study using template prostate mapping biopsies as the reference standard," Prostate Cancer and Prostatic Diseases, vol. 17, no. 1, pp. 40-46, 2014.

[32] K. Chamie, G. A. Sonn, D. S. Finley et al., "The role of magnetic resonance imaging in delineating clinically significant prostate cancer," Urology, vol. 83, no. 2, pp. 369-375, 2014.

[33] S. Rais-Bahrami, M. M. Siddiqui, B. Turkbey et al., "Utility of multiparametric magnetic resonance imaging suspicion levels for detecting prostate cancer," The Journal of Urology, vol. 190, no. 5, pp. 1721-1727, 2013.

[34] P. Rouse, G. Shaw, H. U. Ahmed, A. Freeman, C. Allen, and M. Emberton, "Multi-parametric magnetic resonance imaging to rule-in and rule-out clinically important prostate cancer in men at risk: a cohort study," Urologia Internationalis, vol. 87, no. 1, pp. 49-53, 2011.

[35] P. J. De Visschere, L. Naesens, L. Libbrecht et al., "What kind of prostate cancers do we miss on multiparametric magnetic resonance imaging?" European Radiology, vol. 26, no. 4, pp. 1098-1107, 2015.

[36] N. Arumainayagam, H. U. Ahmed, C. M. Moore et al., "Multiparametric MR imaging for detection of clinically significant prostate cancer: a validation cohort study with transperineal template prostate mapping as the reference standard," Radiology, vol. 268, no. 3, pp. 761-769, 2013.

[37] M. Abd-Alazeez, H. U. Ahmed, M. Arya et al., "The accuracy of multiparametric MRI in men with negative biopsy and elevated PSA level-can it rule out clinically significant prostate cancer?" Urologic Oncology: Seminars and Original Investigations, vol. 32, no. 1, pp. 45-e22, 2014.

[38] K. C. Latchamsetty, L. S. Borden Jr., C. R. Porter et al., "Experience improves staging accuracy of endorectal magnetic resonance imaging in prostate cancer: what is the learning curve?" The Canadian Journal of Urology, vol. 14, no. 1, pp. 3429-3434, 2007.

[39] O. Akin, C. C. Riedl, N. M. Ishill, C. S. Moskowitz, J. Zhang, and H. Hricak, "Interactive dedicated training curriculum improves accuracy in the interpretation of MR imaging of prostate cancer," European Radiology, vol. 20, no. 4, pp. 995-1002, 2010.

[40] H. Cash, K. Günzel, A. Maxeiner et al., "Prostate cancer detection on transrectal ultrasonography-guided random biopsy despite negative real-time magnetic resonance imaging/ultrasonography fusion-guided targeted biopsy: reasons for targeted biopsy failure," BJU International, 2015. 
[41] G. Ploussard, N. Nicolaiew, C. Marchand et al., "Prospective evaluation of an extended 21-core biopsy scheme as initial prostate cancer diagnostic strategy," European Urology, vol. 65, no. 1, pp. 154-161, 2014.

[42] M. Valerio, C. Anele, A. Freeman et al., "Identifying the index lesion with template prostate mapping biopsies," The Journal of Urology, vol. 193, no. 4, pp. 1185-1190, 2015.

[43] A. B. Barqawi, K. O. Rove, S. Gholizadeh, C. I. O’Donnell, H. Koul, and E. D. Crawford, "The role of 3-dimensional mapping biopsy in decision making for treatment of apparent early stage prostate cancer," The Journal of Urology, vol. 186, no. 1, pp. 8085, 2011. 


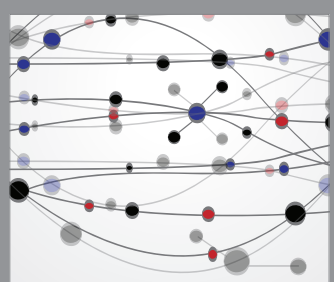

The Scientific World Journal
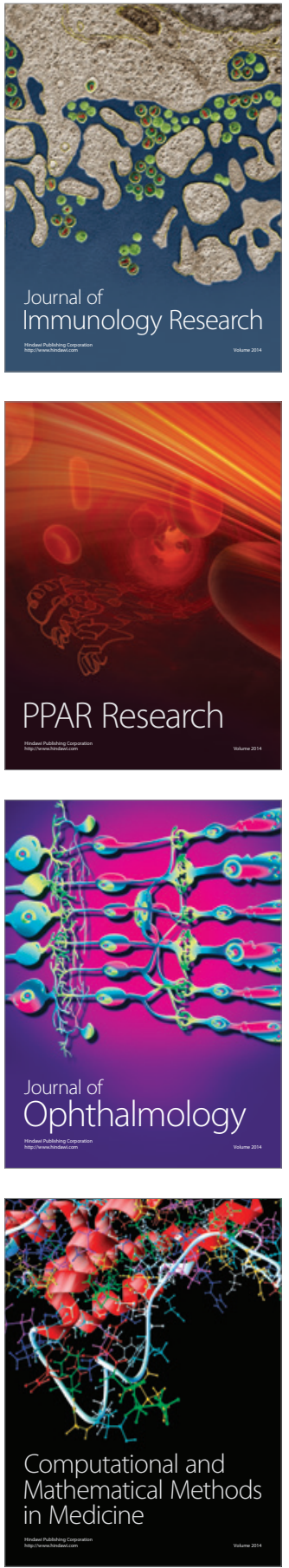

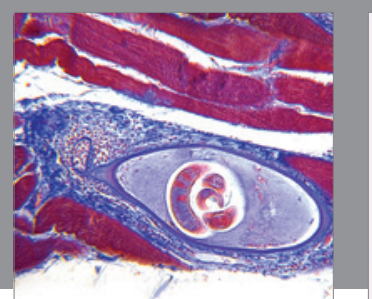

Gastroenterology Research and Practice

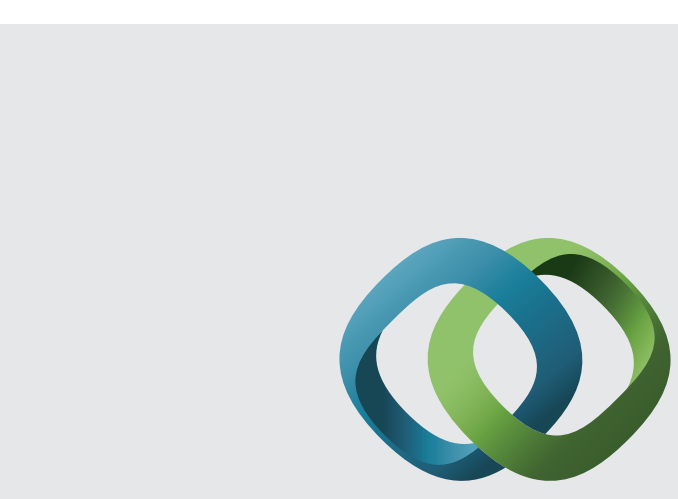

\section{Hindawi}

Submit your manuscripts at

http://www.hindawi.com
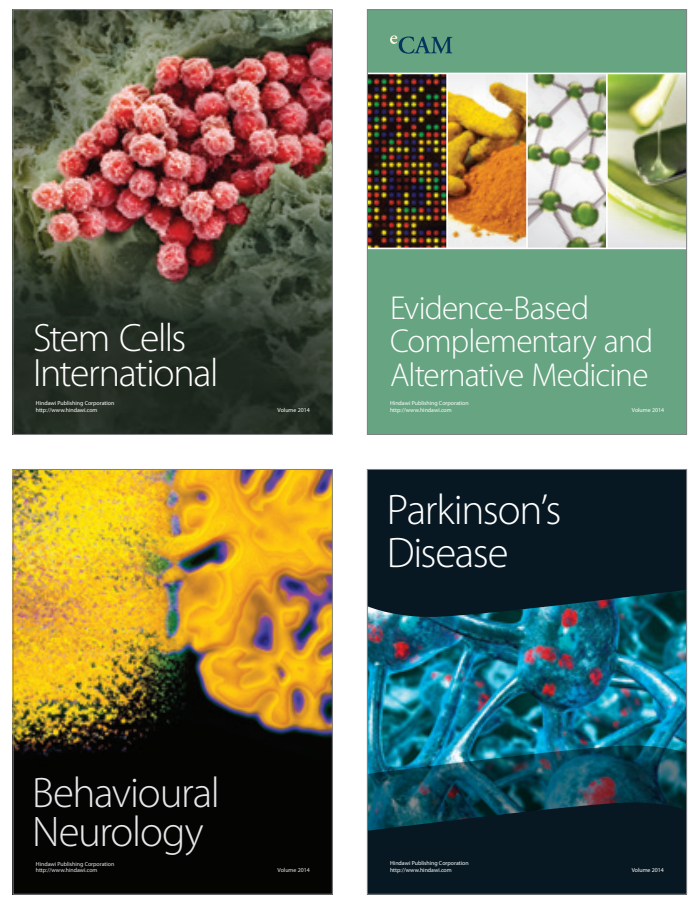
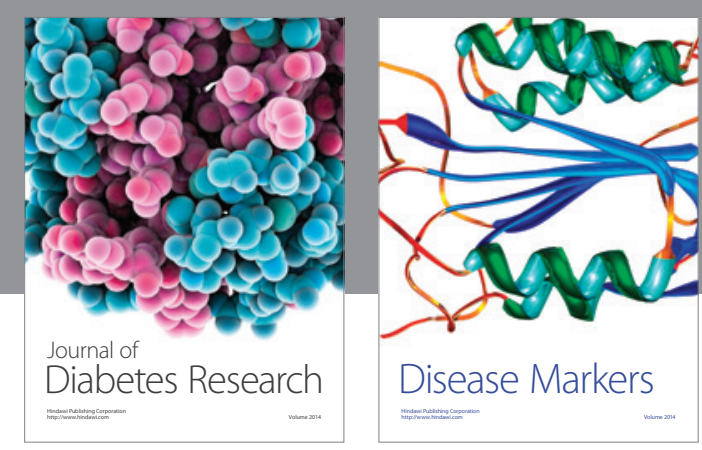

Disease Markers
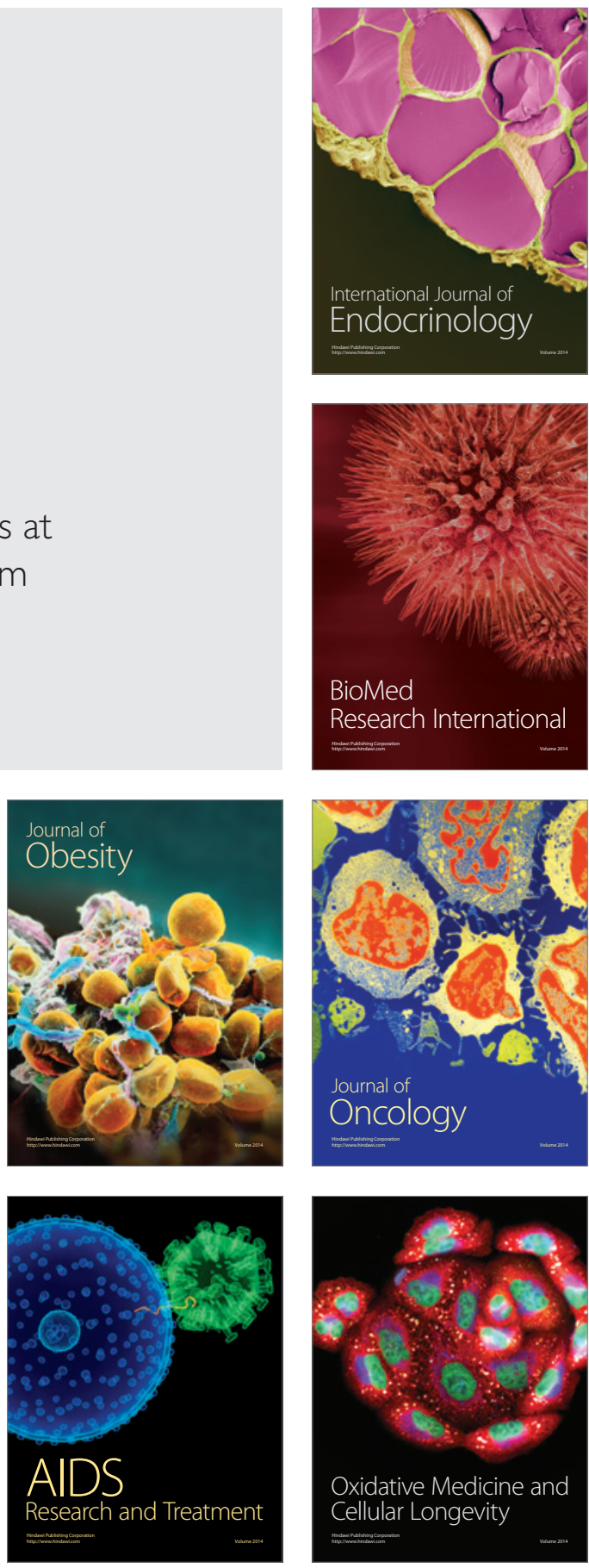